\title{
EFEITO DOS ÁCIDOS HEXENURÔNICOS E DA LIGNINA NO DESEMPENHO DA OZONÓLIZE, EM DIFERENTES pHs DA REAÇÃO
}

\author{
Gustavo Ventorim* \\ Departamento Engenharia Industrial Madeireira, Universidade Estadual Paulista, Rua Geraldo Alckmin, 519, \\ 18409-010 Itapeva - SP, Brasil \\ Jorge Luiz Colodette \\ Departamento Engenharia Florestal, Universidade Federal de Viçosa, 36571-000 Viçosa - MG, Brasil
}

Recebido em 13/4/05; aceito em 27/1/06; publicado na web em 14/6/06

\begin{abstract}
HEXENURONIC ACID AND LIGNIN EFFECTS ON OZONOLYSIS PERFORMANCE IN DIFFERENT REACTION pHs. The effect of $\mathrm{pH}$ on the performance of the ozonolysis stage in pulp production was evaluated for conventional and acid treated brown and oxygen delignified eucalyptus kraft pulps. The objective was to determine separately the effects of lignin and hexenuronic acid on the performance of the ozonolysis stage. The reaction of ozone with hexenuronic acid is less sensitive to $\mathrm{pH}$ than the reaction of ozone with lignin. The efficiency and the selectivity of the reaction of ozone with pulp decreases after removal of hexenuronic acids. Increasing up to 7.0 the $\mathrm{pH}$ during the ozonolysis is viable in the sequence $\mathrm{Z} / \mathrm{D}(\mathrm{EOP}) \mathrm{D}$, resulting in savings of $\mathrm{H}_{2} \mathrm{SO}_{4}(8,5$ $\mathrm{kg} / \mathrm{tsa})$ and $\mathrm{NaOH}(5 \mathrm{~kg} / \mathrm{tsa})$, but is not recommended in the sequence $\mathrm{Z} / \mathrm{ED}(\mathrm{PO})$.
\end{abstract}

Keywords: ozone; brown pulp; oxygen delignification.

\section{INTRODUÇÃOO}

É bem documentado na literatura o fato de que o branqueamento de polpas celulósicas com ozônio deve ser efetuado em $\mathrm{pH}$ na faixa de 2,5-3, já que em valores acima destes ocorre significativa decomposição do ozônio, catalisada por íons $\mathrm{OH}^{-}$, o que resulta em perda de eficiência e seletividade do reagente ${ }^{1-4}$. A perda de eficiência deriva das perdas de ozônio e a perda de seletividade, do ataque aos carboidratos, presentes na polpa celulósica, pelos radicais livres (HOO*, HO*) provenientes da decomposição do ozônio.

As conclusões citadas acima foram obtidas de experimentos e experiências industriais realizados, principalmente, com polpa kraft de fibra longa e de kappa (determinação indireta do teor de lignina e de ácido hexenurônico presentes na polpa de celulose) elevado (30), sendo este número kappa essencialmente constituído de produtos de degradação da lignina. Existem poucos estudos com relação ao efeito do $\mathrm{pH}$ da ozonólise em polpas de fibra curta de baixo número kappa (10), sendo este baixo kappa constituído principalmente de ácidos hexenurônicos (HexA's) ${ }^{5}$. A lignina propaga as reações radicalares quando reage com ozônio ${ }^{6}$, portanto, fica fácil entender o impacto negativo que polpas com níveis altos de lignina sofrem mediante uma reação com ozônio em pH acima do ideal, sendo que neste $\mathrm{pH}$ o ozônio apresenta maior degradação em radicais livres ${ }^{4}$. Entretanto, os ácidos hexenurônicos (Figura 1) não têm o mesmo comportamento da lignina durante o branqueamento com ozônio, podendo não sofrer os mesmos efeitos quando oxidados por este em faixas de $\mathrm{pH}$ mais elevadas.

Estudos cinéticos que avaliaram a reação do ozônio com ácidos hexenurônicos indicam que estes reagem duas vezes mais rápido que a lignina ${ }^{8}$.

Do ponto de vista prático operacional, o branqueamento de polpa com ozônio é mais fácil e mais barato se for efetuado em valores de $\mathrm{pH}$ mais elevados que o considerado ideal $(2,5-3,0)$, já que a polpa que chega a um estágio de ozonólise provém, usualmente, de uma etapa de deslignificação com oxigênio efetuada em meio al-

*e-mail: ventorim@itapeva.unesp.br

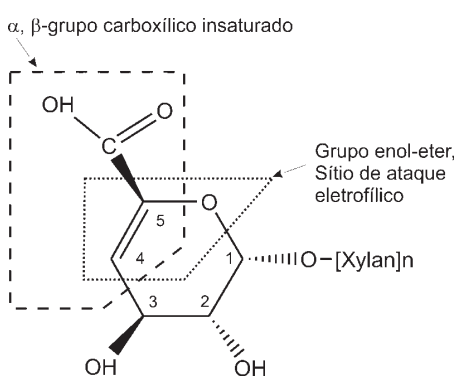

Figura 1. Estrutura do ácido hexenurônico. Adaptada da ref. 7

calino. Assim, a operação da etapa de ozônio em pH neutro ou alcalino certamente requer menor consumo de reagentes para ajuste prévio de $\mathrm{pH}$ e ajuste posterior de $\mathrm{pH}$, no caso da etapa subseqüente ser realizada em $\mathrm{pH}$ também mais elevado.

O objetivo deste estudo foi determinar o efeito dos teores de HexA's e de lignina no pH ideal de operação da ozonólise, visando reduzir o consumo de ácido sulfúrico e hidróxido de sódio. Tal objetivo foi atingido através da determinação do efeito do $\mathrm{pH}$ de reação (faixa de 2,5 a 9,0) na eficiência e seletividade do branqueamento de polpas de eucalipto com ozônio - as que continham níveis variáveis de lignina e de HexA's - em sequiências do tipo Z/ $\mathrm{D}(\mathrm{EOP}) \mathrm{D}$ e $\mathrm{A}_{\mathrm{HT}} \mathrm{Z} / \mathrm{D}(\mathrm{EOP}) \mathrm{D}, \mathrm{Z} / \mathrm{E}$ e $\mathrm{A}_{\mathrm{HT}} \mathrm{Z} / \mathrm{E}$.

\section{PARTE EXPERIMENTAL}

\section{Material}

Foram utilizadas polpas marrons e kraft-O industriais de eucalipto, cujas características são relatadas na Tabela 1 .

\section{Métodos}

Branqueamento com ozônio e dióxido de cloro em média consistência (Z/D)

O branqueamento com ozônio e dióxido de cloro em média 
Tabela 1. Caracterização das polpas

\begin{tabular}{lcc}
\hline Característica da Polpa & Polpa Marrom & Polpa pré-O $\mathrm{O}_{2}$ \\
\hline Número kappa & 17,0 & 9,4 \\
Viscosidade (dm $3 / \mathrm{kg})$ & 1123 & 992 \\
Alvura (\% ISO) & 37,8 & 56,2 \\
HexA's (mmol/kg) & 53,2 & 51,0 \\
HexA's (ud kappa) & 5,3 & 5,1 \\
Lignina solúvel (\%) & 0,62 & 0,78 \\
Lignina Insolúvel (\%) & 1,3 & 0,60 \\
\hline
\end{tabular}

consistência (10\%) foi realizado em um reator/misturador Mark V (Quantum Technologies) acoplado a um compressor de ozônio (Ozone Cart), com amostras de $280 \mathrm{~g}$ (em base seca), nas condições apresentadas na Tabela 2. A polpa foi acidificada com $\mathrm{H}_{2} \mathrm{SO}_{4}$ 2 mol L ${ }^{-1}$ até o $\mathrm{pH}$ desejado e a dosagem pré-estabelecida de ozônio foi injetada no reator através do Ozone Cart. Em seguida, o dióxido de cloro (a produção do dióxido de cloro foi realizada pela adição de ácido sulfúrico $2 \mathrm{~mol} \mathrm{~L}^{-1}$ em clorito de sódio, com isso há liberação de dióxido de cloro que é armazenado em um frasco contendo água) foi adicionado à polpa. Terminada a reação, o ozônio residual, no Ozone Cart, foi coletado em uma solução de KI $5 \%$ e titulado por iodometria conforme $\mathrm{Kraft}^{10}$.

O ozônio consumido pela polpa foi calculado pela diferença entre as concentrações de ozônio aplicada e residual. Terminada a reação, foram extraídas amostras de licor para análises de $\mathrm{pH}$ final e ozônio/dióxido residual, e a polpa foi lavada com $\left(9 \mathrm{~m}^{3} / \mathrm{t}\right.$ de polpa, base seca) água destilada. $\mathrm{O}$ estágio $\mathrm{Z} / \mathrm{D}$ foi efetuado em duplicata.

Os estágios EOP (Extração Oxidativa com Oxigênio e Peróxido), $\mathrm{A}_{\mathrm{HT}}$ (Hidrólise Ácida em alta temperatura) e Z (Ozônio) também foram realizados no equipamento Mark $\mathrm{V}$, enquanto que o estágio $\mathrm{D}$ (Dioxidação) foi realizado em sacos de polietileno. As condições desses tratamentos estão apresentadas na Tabela 2. Para o estágio de extração alcalina as condições pré-estabelecidas foram consistência $=10 \%$, temperatura $=60{ }^{\circ} \mathrm{C}$, tempo $=90 \operatorname{min~e~} \mathrm{pH}_{\text {final }}=11$.

Tabela 2. Condições de branqueamento

\begin{tabular}{lcccccc}
\hline Condições & \multicolumn{5}{c}{ Estágio de Branqueamento } \\
& $\mathrm{A}_{\mathrm{HT}}$ & $\mathrm{Z}$ & $\mathrm{D}$ & $(\mathrm{EOP})$ & $\mathrm{D}$ \\
\hline Consistência $(\%)$ & 12 & 12 & 10 & 12 & 12 \\
Temperatura $\left({ }^{\circ} \mathrm{C}\right)$ & 95 & 55 & 60 & 85 & 84 \\
Tempo (min) & 120 & 2 & 40 & 40 & 60 \\
Pressão inicial $(\mathrm{kPa})$ & - & - & - & 200 & - \\
$\mathrm{H}_{2} \mathrm{O}_{2}(\%) \mathrm{g} / 100 \mathrm{~g}$ de polpa & - & - & - & 0,50 & - \\
$\mathrm{O}_{2}(\%) \mathrm{g} / 100 \mathrm{~g}$ de polpa & - & - & - & 0,60 & - \\
$\mathrm{O}_{3}(\%) \mathrm{g} / 100 \mathrm{~g}$ de polpa & - & 0,40 & - & - & - \\
$\left.\mathrm{NaOH}^{2} \%\right) \mathrm{g} / 100 \mathrm{~g}$ & - & - & 0,80 & 0,80 & - \\
de polpa & & & & & \\
$\mathrm{H}_{2} \mathrm{SO}(\%) \mathrm{g} / 100 \mathrm{~g}$ & 0,80 & 0,18 & - & - & 0,13 \\
de polpa & & & & & \\
$\mathrm{ClO}_{2}(\%) \mathrm{g} / 100 \mathrm{~g}$ de polpa & - & - & 0,684 & - & 0,076 \\
\hline
\end{tabular}

\section{Procedimentos analíticos}

As análises da polpa após o branqueamento foram efetuadas seguindo os procedimentos analíticos como: número kappa, norma TAPPI om 245, viscosidade, norma TAPPI T230 om 82, alvura, norma TAPPI T525 om 86, reversão de alvura 4 h, $105{ }^{\circ} \mathrm{C}, 0 \%$ umidade relativa, após acondicionamento das folhas por $4 \mathrm{~h}$ em sala climatizada, lignina insolúvel, lignina solúvel ${ }^{9}$, titulações dos reagentes e residuais de branqueamento ${ }^{10}$, OX (halogênios orgâni- cos presente na polpa), norma SCAN-W9:89 e TOC (carbono orgânico total) do efluente, norma Standard Methods. Todas as análises foram efetuadas em duplicata. As análises dos ácidos hexenurônicos foram feitas por cromatografia (HPLC), nas seguintes condições: fase estacionária: coluna Shim pack SCR - 102H; fase móvel: $\mathrm{H}_{2} \mathrm{SO}_{4} 5,0 \mathrm{mmol} / \mathrm{L}$; fluxo: $1,5 \mathrm{~mL} / \mathrm{min}$; temperatura: $60{ }^{\circ} \mathrm{C}$; detector: UV $254 \mathrm{~nm}$.

\section{Cálculo do equivalente de oxidação (OXE)}

Para cálculo do OXE foram seguidos os seguintes passos: calculou-se a massa molecular do composto (ex. $\mathrm{ClO}_{2}$ ); determinouse a quantidade de elétrons transferida na reação do composto em questão (5); dividiu-se a massa molecular pelo número de elétrons transferidos, determinando-se o número de equivalentes do composto $(67,5 / 5)$.

O OXE é o produto da divisão de $1 \mathrm{~kg}$ do composto em $\mathrm{g}$, pelo número de equivalentes $(1000 / 13,5=74,07)$. Para se determinar o OXE da sequiência de branqueamento, basta multiplicar o OXE de cada composto pela carga aplicada $(\mathrm{kg} / \mathrm{t})$ em cada estágio.

\section{RESULTADOS E DISCUSSÃO}

Efeito do $\mathrm{pH}$ do estágio $\mathrm{Z}$ no branqueamento da polpa prédeslignificada com oxigênio (pré- $\mathrm{O}_{2}$ ) pela seqüência $\mathrm{Z} / \mathrm{D}(\mathrm{EOP}) \mathrm{D}$

Um sumário do efeito do $\mathrm{pH}$ do estágio $\mathrm{Z}$ no desempenho do branqueamento da polpa pré- $\mathrm{O}_{2}$ pela sequiência $\mathrm{Z} / \mathrm{D}(\mathrm{EOP}) \mathrm{D}$ é apresentado nas Figuras 2, 3 e 4.

Verifica-se que os consumos totais de ácido sulfúrico e de hidróxido de sódio decresceram $8,5 \mathrm{~kg} / \mathrm{tsa}(\sim 80 \%)$ e $5 \mathrm{~kg} / \mathrm{tsa}$ ( $30 \%)$, respectivamente, com a elevação do $\mathrm{pH}$ da ozonólise de 2,5 para 7,0. Essa economia de reagentes se deve à menor oscilação do pH entre os estágios Z/D e (EOP), quando o estágio $\mathrm{Z}$ foi efetuado em pH 7 (Figura 2).

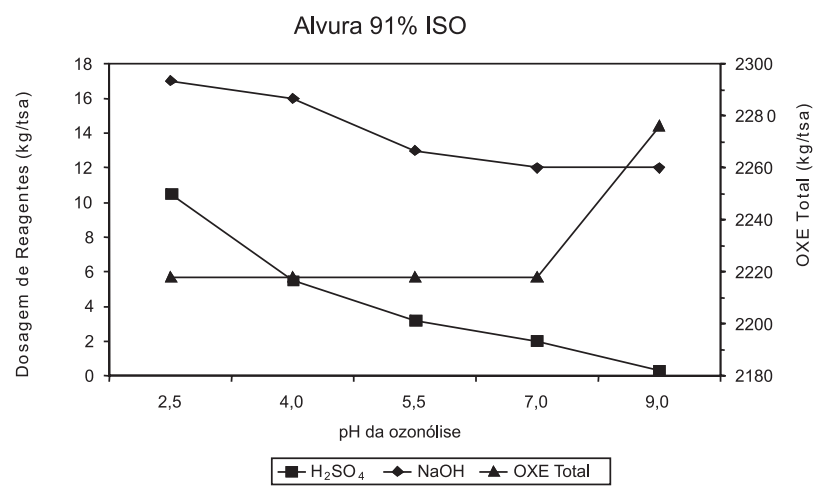

Figura 2. Efeito do $\mathrm{pH}$ da ozonólise no consumo de $\mathrm{NaOH}, \mathrm{H}_{2} \mathrm{SO}_{4}$ e oxidantes pela seqüência $\mathrm{Z} / \mathrm{D}(\mathrm{EOP}) \mathrm{D}$ - polpa pré- $\mathrm{O}_{2}$

Surpreendentemente, o consumo total de oxidantes $\left(\mathrm{O}_{2}, \mathrm{O}_{3}, \mathrm{ClO}_{2}\right)$ requeridos para se obter alvura final de $91 \%$ ISO não foi influenciado pelo $\mathrm{pH}$ da ozonólise até o valor de 7,0, contrariamente ao que tem sido amplamente divulgado na literatura especializada ${ }^{1}$ (Figura 2). O número kappa é constituído basicamente de lignina e de ácidos hexenurônicos, sendo que a polpa investigada apresentava aproximadamente cinco unidades de número kappa devidas aos ácidos hexenurônicos, os quais reagem rapidamente com ozônio - velocidade de reação de $130 \mathrm{mmol} \mathrm{HexA}$ 's $/ \mathrm{kg}$ polpa $/ \mathrm{min}^{8}$. Essa alta velocidade de reação explica a pouca importância do $\mathrm{pH}$. 
Uma avaliação do kappa da polpa medido após (EOP) indica ligeira perda de eficiência da ozonólise com o aumento do $\mathrm{pH}$ de 2,5 para 9, que resultou em um aumento do kappa (EOP) de 1,3 para 1,9 (Figura 3). Também, deve ser notado que os teores de lignina solúvel e de HexA's, medidos após a ozonólise, tenderam a aumentar ligeiramente com o aumento do $\mathrm{pH}$ da ozonólise (Figura 3).

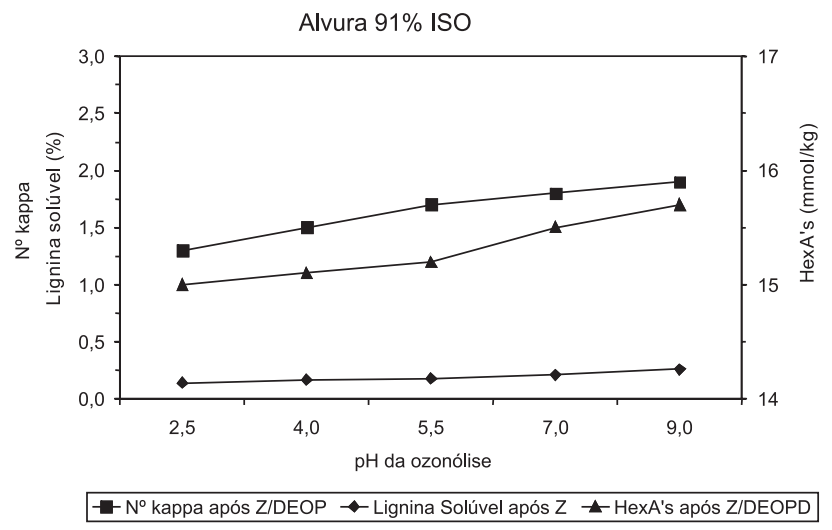

Figura 3. Efeito do $p H$ da ozonólise no número kappa após $Z / D(E O P)$, no teor de lignina solúvel após $Z$ e no teor de HexA's após $Z / D(E O P) D$ - polpa pré- $\mathrm{O}_{2}$

Os valores de reversão de alvura e de OX da polpa branqueada não foram influenciados significativamente pelo $\mathrm{pH}$ da ozonólise. Por outro lado, a viscosidade final da polpa decresceu ligeiramente com o aumento do $\mathrm{pH}$, havendo uma perda de $67 \mathrm{dm}^{3} / \mathrm{kg}$ com a elevação do $\mathrm{pH}$ de 2,5 para 9 (Figura 4). Esta perda é oriunda principalmente da formação de radicais livres durante a ozonólise com o aumento de $\mathrm{pH}$.

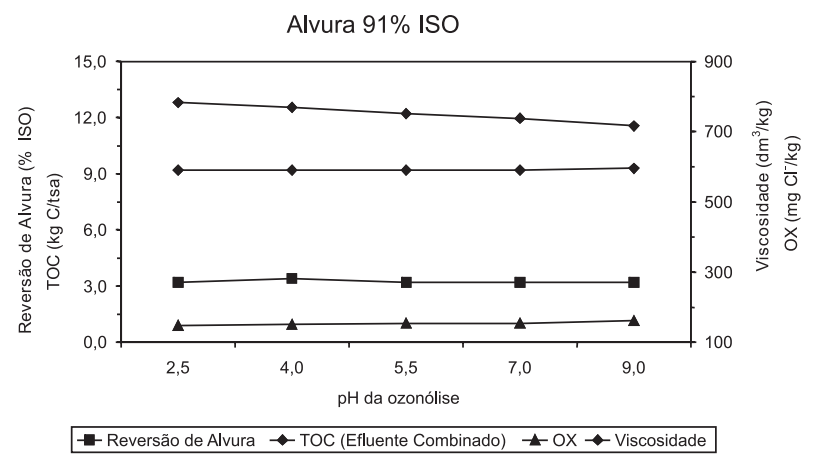

Figura 4. Efeito do $p H$ da ozonólise na reversão de alvura $\left(\Delta R_{457}\right)$, na viscosidade final e teor de OX da polpa branqueada e no TOC do efluente combinado da seqüência Z/D(EOP)D - polpa pré- $O_{2}$

Estudos realizados por outros investigadores com polpa de fibra longa também mostraram perdas de eficiência e seletividade da ozonólise com o aumento do $\mathrm{pH}$ de reação ${ }^{11}$, porém elas foram muito mais severas que as verificadas aqui. A razão para essas diferenças pode estar relacionada ao teor de HexA's da polpa utilizada por esses investigadores.

A sequiência $A_{H T} Z / D(E O P) D$ apresentou as mesmas tendências mostradas para a seqüência $\mathrm{Z} / \mathrm{D}(\mathrm{EOP}) \mathrm{D}$.

\section{Impacto do conteúdo de HexA's da polpa no $\mathrm{pH}$ ótimo da ozonólise}

Os resultados da Figura 5 mostram o efeito do pH da ozonólise efetuada em duas amostras de polpa marrom - uma normal e outra tratada com ácido $\left(\mathrm{A}_{\mathrm{HT}}\right)$ para remoção parcial dos HexA's - sendo a ozonólise seguida de extração alcalina simples em vez de dioxidação.

Verifica-se na Figura 5 que a remoção parcial dos HexA's da polpa por um tratamento ácido resulta em decréscimo substancial da eficiência desta etapa, fato este explicado pela grande preferência do ozônio pelos HexA's da polpa. A velocidade de reação do ozônio diante dos HexA's é duas vezes maior que com a lignina ${ }^{8}$. Quando estes ácidos estão em menor quantidade na polpa, o ozônio tende a reagir mais com os carboidratos, reduzindo assim a seletividade da ozonólise ${ }^{12}$. O autor explica o fenômeno pela maior formação de radicais livres no sistema quando o ozônio reage com uma polpa contendo alta relação lignina/HexA's, isto é, uma polpa pré-tratada com ácido. No entanto, a velocidade da reação da lignina diante do ozônio não foi influenciada pela presença dos HexA's na polpa $^{8}$. Note que a eficiência da ozonólise é medida pela redução do número kappa por $\mathrm{kg}$ de $\mathrm{O}_{3}$ aplicado ao sistema (4 kg/tsa), e a seletividade, em unidades de número kappa perdidas por unidade de viscosidade removida.

Contrariamente ao que fora notado anteriormente, para o caso da ozonólise seguida de dioxidação, processos $\mathrm{Z} / \mathrm{D}(\mathrm{EOP})$ e $\mathrm{A}_{\mathrm{HT}} \mathrm{Z} /$ D(EOP) (Figuras 2 a 4), observa-se na Figura 5 que a eficiência e a seletividade da ozonólise seguida de simples extração, processos $\mathrm{Z} / \mathrm{E}$ e $\mathrm{A}_{\mathrm{HT}} \mathrm{Z} / \mathrm{E}$, decrescem significativamente com o aumento do $\mathrm{pH}$ da reação, sendo o efeito negativo mais pronunciado na polpa tratada com ácido, isto é, na polpa contendo menor teor de HexA's. O aumento do $\mathrm{pH}$ de 2,5 para 7,0 decresceu a eficiência da ozonólise de 1,33 para 1,18 na polpa marrom $(\sim 11 \%)$ e de 1,03 para 0,63 na polpa marrom previamente tratada com ácido ( 40\%). Tendência similar foi observada no caso da seletividade. Portanto, não se recomenda operar a ozonólise em valores de $\mathrm{pH}$ acima do ideal (2,5$3,0)$, se esta etapa for seguida de extração alcalina simples em vez de dioxidação, especialmente para polpas com baixos teores de HexA's (ex. polpas tratadas com um estágio de hidrólise ácida a quente).

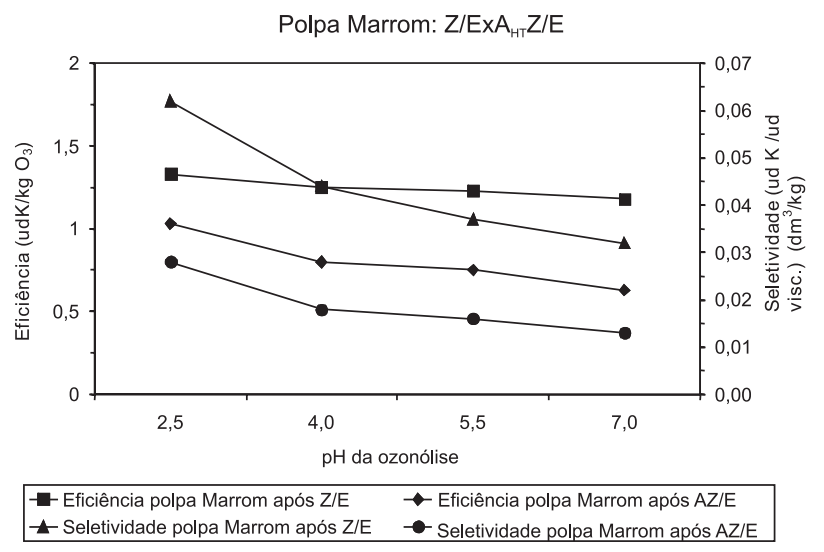

Figura 5. Efeito do pH nos resultados da ozonólise, medida após extração simples (10\% cst, $60^{\circ} \mathrm{C}, 90 \mathrm{~min}, \mathrm{pH} \mathrm{11,0)}$ ), de uma polpa marrom de eucalipto não-tratada $(Z / E)$ e tratada com ácido $\left(A_{H T} Z / E\right)$

$\mathrm{O}$ fato de o aumento do $\mathrm{pH}$ ter efeito muito negativo na seletividade da ozonólise quando esta é seguida de extração simples, e pouco negativo quando esta é seguida de uma dioxidação, pode ser explicado pela elevada formação de carbonilas na polpa durante a etapa de ozonólise. Nos casos de $\mathrm{Z} / \mathrm{E}$ e $\mathrm{A}_{\mathrm{HT}} \mathrm{Z} / \mathrm{E}$ as carbonilas geradas nos carboidratos durante a ozonólise são eliminadas na etapa 
de extração $(\mathrm{pH} \text { alto })^{11}$, tendo como conseqüência uma redução significativa da viscosidade - a eliminação de carbonilas em meio alcalino é sempre acompanhada de quebra da cadeia de celulose. Quando a ozonólise é seguida de dioxidação, como nos processos $\mathrm{Z} / \mathrm{D}(\mathrm{EOP})$ e $\mathrm{A}_{\mathrm{HT}} \mathrm{Z} / \mathrm{D}(\mathrm{EOP})$, tais carbonilas são oxidadas pelo dióxido de cloro antes da (EOP), o que reduz o risco de perda de viscosidade na etapa alcalina.

A eficiência da ozonólise tende a ser maior quando é seguida de extração simples em vez de dioxidação, devido à formação de sais de ácidos inorgânicos pelo álcali das estruturas de lignina oxidadas pelo ozônio. A eficiência da formação de sais de ácidos inorgânicos é tanto maior quanto mais baixo for o $\mathrm{pH}$ da ozonólise. Por conseguinte, quando o $\mathrm{pH}$ da ozonólise é elevado de 2,5 para 7,0 , a eficiência desta etapa, medida após $\mathrm{Z} / \mathrm{E}$ ou $\mathrm{A}_{\mathrm{HT}} \mathrm{Z} / \mathrm{E}$, decresce significativamente. No caso dos processos Z/D(EOP) e $\mathrm{A}_{\mathrm{HT}} \mathrm{Z} / \mathrm{D}(\mathrm{EOP})$ não ocorre a formação de sais ácidos inorgânicos após a ozonólise, pois a etapa seguinte é de dioxidação, uma etapa ácida. Por isso, o efeito do $\mathrm{pH}$ da ozonólise é menos importante nesse caso. Deve-se notar que a eficiência global da ozonólise é sempre muito maior se esta etapa for seguida de extração alcalina.

\section{Impacto do conteúdo de lignina da polpa no $\mathrm{pH}$ ótimo da ozonólise}

Os resultados da Figura 6 mostram o efeito do pH na redução do número kappa e do conteúdo de ácido hexenurônico, medidos após o estágio $\mathrm{Z} / \mathrm{D}(\mathrm{EOP})$, quando efetuado em duas amostras de polpa, uma pré- $\mathrm{O}_{2}$ com 4,3 unidades de kappa devido à lignina $\mathrm{e}$ outra marrom contendo 11,7 unidades de kappa devido à lignina. $\mathrm{O}$ conteúdo de HexA's das amostras de polpa pré- $\mathrm{O}_{2}$ e marrom era similar - respectivamente, 51 e $53,2 \mathrm{mmol} / \mathrm{kg}$ de polpa.

Verifica-se na Figura 6 que, muito embora os teores de HexA's das polpas pré- $\mathrm{O}_{2}$ e marrom fossem similares, a eficiência e a seletividade da ozonólise foram maiores para a polpa marrom, devido ao seu maior teor de lignina. A eficiência para a polpa marrom variou de 0,90 a 0,92 , enquanto a da polpa pré- $\mathrm{O}_{2}$ ficou na faixa de 0,70 a 0,75 . Por outro lado, a seletividade para a polpa marrom foi melhor, variando de 15,3 a 17,4 , ao passo que a da polpa pré- $\mathrm{O}_{2}$ variou de 16,2 a 24,2 . Deve-se lembrar que menores valores de seletividade significam maiores seletividades, visto que esta é expressa em unidades de viscosidade perdidas por unidade

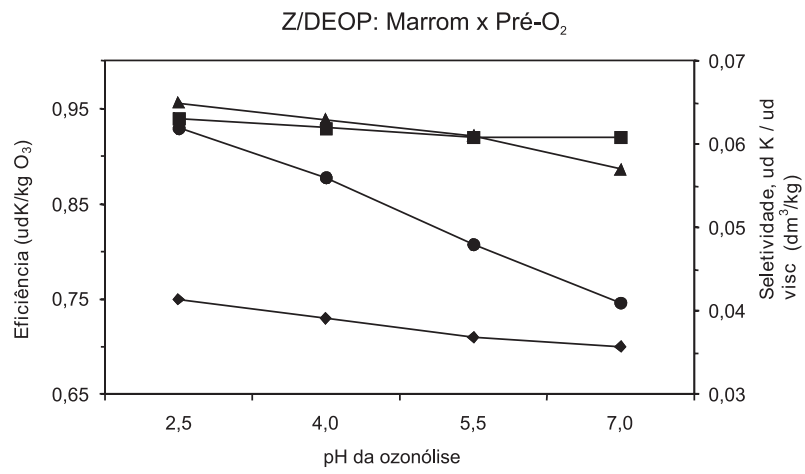

- Eficiência polpa Marrom após Z/DEOP - Eficiência polpa Pré- $\mathrm{O}_{2}$ após Z/DEOP —- Seletividade polpa Marrom após Z/DEOP —- Seletividade polpa Pré- $\mathrm{O}_{2}$ após Z/DEOP

Figura 6. Resultados de eficiência e seletividade da ozonólise, medidos após $\mathrm{Z} / \mathrm{D}(\mathrm{EOP})$, de uma polpa pré- $\mathrm{O}_{2}$ e outra marrom de eucalipto de número kappa removido. Note que, nesse caso, a eficiência da ozonólise foi medida pela redução do número kappa por $\mathrm{kg}$ de $\mathrm{ClO}_{2}$ ativo aplicado ao sistema. No caso da polpa pré- $\mathrm{O}_{2}, \mathrm{o} \mathrm{ClO}_{2}$ ativo total aplicado foi de $10,84 \mathrm{~kg} / \mathrm{tsa}\left(4 \mathrm{~kg} / \mathrm{tsa}\right.$ de $\mathrm{O}_{3}$ aplicado em Z mais $6,84 \mathrm{~kg} / \mathrm{tsa}$ de $\mathrm{ClO}_{2}$ aplicado em D). No caso da polpa marrom, o total aplicado foi de 16,2 (4 kg/tsa de $\mathrm{O}_{3}$ aplicado em Z mais $12,2 \mathrm{~kg} /$ tsa de $\mathrm{ClO}_{2}$ aplicado em D).

Observa-se também na Figura 6 que a eficiência e a seletividade da ozonólise decrescem ligeiramente com o aumento do $\mathrm{pH}$ da reação, sendo o efeito negativo mais pronunciado na polpa pré- $\mathrm{O}_{2}$, isto é, naquela com menor teor de lignina. $\mathrm{O}$ aumento do $\mathrm{pH}$ de 2,5 para 7,0 decresceu a eficiência da ozonólise de 0,75 para 0,70 na polpa pré- $\mathrm{O}_{2}(\sim 7 \%)$ e de 0,94 para 0,92 na polpa marrom $(\sim 2 \%)$. Tendência similar foi observada no caso da seletividade. Deve ser notado que o efeito do $\mathrm{pH}$ na eficiência e na seletividade (na faixa de 2,5-7,0) foi pouco significativo, sendo o seu impacto tecnológico ao final do branqueamento de menor importância.

A seqüência $A_{H T} Z / D(E O P)$, contendo o estágio $A_{H T}$, foi também avaliada quanto ao efeito do $\mathrm{pH}$ no desempenho da ozonólise, medida após o estágio da (EOP), quando efetuada em duas amostras de polpa, uma pré- $\mathrm{O}_{2}$ com 4,3 unidades de kappa devido à lignina e outra marrom contendo 11,7 unidades de kappa devido à lignina. No que tange ao efeito do $\mathrm{pH}$ da ozonólise, as mesmas tendências discutidas anteriormente, medidas após Z/D(EOP), são válidas para as polpas previamente tratadas com um estágio de hidrólise ácida, i.e., medidas após $\mathrm{A}_{\mathrm{HT}} \mathrm{Z} / \mathrm{D}(\mathrm{EOP})$. No entanto, as eficiências da ozonólise são menores nas polpas tratadas com ácido em relação àquelas não-tratadas. E ainda, as diferenças na eficiência da ozonólise para polpas marrom e pré- $\mathrm{O}_{2}$ são muito mais pronunciadas nas amostras tratadas com o estágio ácido.

\section{CONCLUSÕES}

O aumento do $\mathrm{pH}$ da ozonólise de 2,5 para 7,0 é justificável para sequiências do tipo Z/D(EOP)D. A reação do ozônio com os HexA's requer menor acidez que com a lignina. A remoção dos ácidos hexenurônicos da polpa de celulose diminui a eficiência e seletividade da ozonólize. Para a sequiência Z/D(EOP)D a elevação do $\mathrm{pH}$ de 2,5 para 7,0 reduz o consumo de ácido sulfúrico em 8,5 $\mathrm{kg} / \mathrm{tsa}$ e de soda em $5 \mathrm{~kg} / \mathrm{tsa}$.

\section{REFERÊNCIAS}

1. Shackford, L. D.; Miller, W. J.; Minami, S.; International Workshop, São Paulo, Brasil, 1995.

2. Bouchard, J.; Nugent, H. M.; Berry, R.; Tappi J. 1995, 1, 74.

3. Liebergott, N.; van Lierop, B.; Skothos, A.; Tappi J. 1992, 1, 145

4. Colodette, J. L.; Singh, U. P.; Ghosh, A. K.; Pulp Paper Journal 1993, 6, 139.

5. Costa, M. M.; Colodette, J. L. ; International Pulp Bleaching Conference, Washington, DC., USA, 1996.

6. Jacobson, B.; Lindblad, P.; Nilvebrant, N.; International Pulp Bleaching Conference, Estocolmo, Suécia, 1991.

7. Jiang, Z.; van Lierop, B. V.; Berry, R.; Tappi J. 2000, 1, 175.

8. Ventorim, G.; Tese de Doutorado, Universidade Federal de Viçosa, Brasil, 2004.

9. Sarkanem, K. V.: Ludwig, C. H.; Lignins, Occurrence, formation, structure and reations, John Wiley and Sons: New York, 1971.

10. Kraft, P.; Pulp \& Paper Manufacture, $2^{\text {nd }}$ ed., McGraw-Hill Book Company: New York, 1967.

11. Dence, C. W.: Reeve, D. W.; Pulp bleaching - principles and practive, Tappi: Atlanta, 1996.

12. Ragnar, M.; Ph. D. Thesis, KTH, USA, 2000. 